\title{
Aspirin and death in Covid-19 A systematic review and meta-analysis
}

\author{
Nicholas Moore (NO NEW ASSIGNMENTS) ${ }^{1}$, Nicolas Thurin ${ }^{1}$, Pauline Bosco-Lévy ${ }^{1}$, \\ Patrick Blin ${ }^{1}$, and cecile Droz ${ }^{1}$ \\ ${ }^{1}$ University of Bordeaux
}

August 27, 2021

\begin{abstract}
Thrombotic events are common during COVID-19 infection. Aspirin might be beneficial. Objective: Systematic review and meta-analysis of deaths in users and non-users of aspirin. Data sources: Pubmed Medline, Google scholar, Clinicaltrials.gov, Cochrane, to June 8, 2021, Study selection: Studies providing adjusted or matched evaluation of association of exposure to aspirin and death in COVID-19 patients were included. Data extraction and synthesis: Data were used as published, as Odds ratio, hazard ratio or relative risks and $95 \% \mathrm{CI}$ from which $\log (\mathrm{OR})$ and SE were recalculated. These were entered in an inverse variance odds ratios random-effects model, using RevMan 5.4 (the Cochrane Collaboration). Main outcomes and measure: The prespecified outcome studied was death. Results: Nine studies ( 8 observational, one interventional) included 14989 patients exposed to aspirin and 15857 unexposed. Overall Odds Ratio of death in aspirin exposed patients in a random effects model was $0.63,95 \%$ confidence interval [0.40-0.99], I2 94\%. Using a fixed-effect model did not change much the result (0.76 [0.710.81], removing the Recovery trial (OR 0.43 [0.38-0.49], I271\%, or the two largest studies (0.66 [0.47-0.93], I2 38\%) reduced heterogeneity without materially altering the results. The funnel plot showed no evident publication bias Conclusion: this meta-analysis suggests that the use of aspirin may be associated with a lower risk of death in COVID-19. Considering the results of the Recovery Study, it would appear preferable to continue aspirin in patients who have a non-covid indication, but possibly useless to add it if they don't.
\end{abstract}

June 1st, 2021

Aspirin and death in Covid-19

A systematic review and meta-analysis

Nicholas Moore MD, PhD, Nicolas Thurin PharmD, PhD, Pauline Bosco-Levy, MD, PhD, Patrick Blin MD, Cecile Droz-Perroteau PhD

Bordeaux PharmacoEpi, Univ. Bordeaux, Inserm CIC1401, Bordeaux, France

Correspondance to Nicholas Moore,

Université de Bordeaux

Rue Leo Saignat, 33076 Bordeaux Cedex, France

Nicholas.moore@u-bordeaux.fr

Word Count:

Key points:

Question: Because COVID-19 is associated with thrombotic events, might aspirin be beneficial? 
Findings: from a meta-analysis of eight observational studies and one open randomized trial providing adjusted or matched results of exposure to aspirin in COVID-19, the random-effects odds ratio for death in aspirin users compared to non-users was $0.63,95 \%$ confidence interval [0.40-0.99], $\mathrm{I}^{2} 94 \%$. Sensitivity analyses were consistent.

Conclusion: Exposure to aspirin was associated with a lower risk of dying in COVID-19 patients. This would warrant further randomized clinical trials.

Abstract:

: Thrombotic events are common during COVID-19 infection. Aspirin might be beneficial.

Objective: Systematic review and meta-analysis of deaths in users and non-users of aspirin.

Data sources: Pubmed Medline, Google scholar, Clinicaltrials.gov, Cochrane, to June 8, 2021,

Study selection: Studies providing adjusted or matched evaluation of association of exposure to aspirin and death in COVID-19 patients were included.

Data extraction and synthesis: Data were used as published, as Odds ratio, hazard ratio or relative risks and 95\% CI from which $\log (\mathrm{OR})$ and SE were recalculated. These were entered in an inverse variance odds ratios random-effects model, using RevMan 5.4 (the Cochrane Collaboration).

Main outcomes and measure: The prespecified outcome studied was death.

Results: Nine studies (8 observational, one interventional) included 14989 patients exposed to aspirin and 15857 unexposed. Overall Odds Ratio of death in aspirin exposed patients in a random effects model was $0.63,95 \%$ confidence interval [0.40-0.99], $\mathrm{I}^{2} 94 \%$. Using a fixed-effect model did not change much the result (0.76 [0.71-0.81], removing the Recovery trial (OR 0.43 [0.38-0.49], $\mathrm{I}^{2} 71 \%$, or the two largest studies $(0.66$ [0.47-0.93], $\left.\mathrm{I}^{2} 38 \%\right)$ reduced heterogeneity without materially altering the results. The funnel plot showed no evident publication bias

Conclusion: this meta-analysis suggests that the use of aspirin may be associated with a lower risk of death in COVID-19. Considering the results of the Recovery Study, it would appear preferable to continue aspirin in patients who have a non-covid indication, but possibly useless to add it if they don't.

Introduction

COVID-19, the clinical manifestation of SARS-COV2 infection, is often accompanied by thrombotic events that contribute to the severity of the symptoms, suggesting a possible benefit from aspirin. (1-3) A previous meta-analysis had found no effect of aspirin on mortality in COVID-19, but included only 3 observational studies, and the statistical analysis did not take into account adjusted results,(4) whereas aspirin users would be expected to be older and with more cardiovascular diseases associated with severe COVID-19 outcomes. Another more recent meta-analysis included 6 of the studies we had already selected, and found a decreased risk of death in patients prescribed aspirin, with an OR of $0.46,95 \% \mathrm{CI}[0.35-0.61]$. (5) We therefore updated the meta-analyses, including more studies, and taking into account only adjusted results.

Problem definition: What is the effect of exposure to aspirin on mortality in COVID-19?

Hypothesis statement: Aspirin might have a favorable effect in COVID-19

Methods

Study outcome of interest was death, as reported.

Exposure of interest was aspirin, at any time or dose.

Data sources: Pubmed Medline, Clinicaltrials.gov, and Google scholar were queried by the authors for studies mentioning COVID-19 in the title, and aspirin in any field. We also scanned the references of the retrieved 
papers, of two meta-analyses $(4,5)$ and of other papers discussing the role of aspirin in the treatment of COVID-19 (1-3, 6-8). Medline was queried with EndNote 20 (Clarivate analytics).

Study selection: Studies were first selected on their title and abstracts indicating the presence of study results. Full text was retrieved for all selected studies and analyzed for studies with clearly described methodology, providing numbers of patients receiving aspirin and controls, the data analysis method, and numerical results including adjusted and/or propensity score matched results, with Odds ratios, hazard ratios or relative risks, and confidence intervals, from which $\log (\mathrm{OR})$ and $\mathrm{SE}$ were recomputed. Because aspirin use is indicated in cardiovascular disease that increase mortality, only studies reporting adjusted or matched analyses including age and concomitant diseases were included. Authors were contacted if there were discrepant results. Studies concerning children were not included, because of negligible mortality. (9)

Exposure was aspirin at any time or dose, prior to inclusion in the study and present at inclusion, considering aspirin long-lasting action on platelets. Outcome was death in aspirin users and non-users. Outcomes were used as is from the publications without attempting reascertainment.

Statistical analysis: These data were input into RevMan 5.4 ((Review Manager (RevMan), Version 5.4), The Cochrane Collaboration, 2020), and analyzed using inverse variance odds ratios in a random effects model. Heterogeneity was assessed with $\mathrm{I}^{2}$ as provided by the analysis program. Sensitivity analyses including testing a fixed effects model and repeating the analysis after excluding the largest studies so that heterogeneity was $0 \%$. A funnel plot was used to check for possible publication bias.

Results.

On June 6th ${ }^{\text {th }}$, 2021, of 98155 papers in Medline mentioning COVID-19 in the title, 87 mentioned aspirin in the title or other fields. Death or mortality were mentioned in respectively 14 ad 23 of these papers.

In Clinicaltrials.gov 20 studies were identified as involving aspirin and COVID-19, none of which provided any usable data: One had been withdrawn, 2 were not yet recruiting, 11 were still recruiting, one was active not recruiting, Five studies are indicated as completed, but provided no usable information. (NCT04425863, NCT04368370 (10), NCT04757792, NCT04518735, NCT04492501)

Google Scholar provided one further study (11)

At the time this paper was written, the results of Recovery, a large randomized open-label study, were provided and added to the analysis. (12)

Nine studies provided usable data. (Table 1) One paper studying the effect of a 5-Drug regimen including aspirin was not retained because the effect of the individual drugs could not be identified. (7)

In total, nine studies were included in this analysis (Table 2), representing 14989 patients exposed to aspirin and 15857 unexposed.

In 4 studies, users and non-users were matched using propensity scores, and further adjusted (13-16). In one study the actual number of deaths in each arm was not given, only the final adjusted odd ratio and confidence intervals. (14) In Recovery (12), treatment allocation was random, but the study was open-label and no placebo was used.

Meta-analysis of these studies using a random effect model showed that the use of aspirin was associated with lower mortality (OR 0.63, 95\% CI [0.40-0.99]) (figure 1), with much heterogeneity ( $\mathrm{I}^{2} 94 \%, \mathrm{p}=0.00001$ ). With a fixed effect model, the odds ratio was 0.76[0.71-0.81]. (Figure 2)

Removing Recovery study (12) results in a lower Odds Ratio $(0.43$ [0,38-0.49]) with still meaningful heterogeneity $\left(\mathrm{I}^{2}=71 \%\right)$ (figure 3 ) Removing the two largest studies, Recovery and Osborne et al (15) did not meaningfully affect the result (OR $0.66[0.47-0.93])$ but reduced heterogeneity $\left(\mathrm{I}^{2}=38 \%, \mathrm{p}=0.14\right)$. (Figure 4)

The funnel plot found no obvious publication bias (Figure 5).

Discussion: 
We found in this meta-analysis of observational studies and one open randomized trial, (12), that exposure to aspirin was associated with a lower risk of dying than non-use. The sensitivity analyses did not alter the results nor did the inclusion or not of the Recovery trial. This was not unexpected, in a disease that seems to have a strong thrombotic component. The result is consistent with the expected effects of aspirin. $(2,17)$ It appears relatively robust with about 15000 patients in either treatment arm.

A previous meta-analysis (4) included only 3 studies, $(11,18,19)$ and used the raw estimates of odds ratios without taking adjustments or matching into account. The odds ratio reported for the meta-analysis was $1.14[0.84-1.50]$

The other meta-analysis (20) included adjusted values from 6 studies $(11,13-15,19,21)$ It found an Odds ratio of 0.46 [0.35-0.61], lower than the one we found, probably because of the additional studies we included $(12,16,18)$

This meta-analysis included mostly observational studies, so that despite the intrinsic quality of the individual studies, the result is at best tentative and will need to be confirmed by proper clinical trials, a number of which are under way, as indicated in the clinicatrials.gov registry, but not completed yet.

Because aspirin is usually given to prevent cardiovascular disorders, patients routinely treated with aspirin will be at higher risk of dying. (4) For this reason we only considered studies that provided adjusted or propensity score matched studies, and the one open-label randomized study, Recovery.

The open-label platform trial Recovery (12) has prepublished that random attribution of aspirin did not improve the outcomes of patients with COVID-19, in patients with no previous use of aspirin and no formal indication or contra-indication for aspirin. Without Recovery the result of the meta-analysis show a lower event rate in patients using aspirin, including Recovery in the analysis did change the results, the global OR going from 0.43 [0.38-0.49-0.89] to 0.76 [0.71-0.81] for the fixed effects model. Of the many reasons that might explain the apparent lack of effect of aspirin in Recovery, the main may be that patients randomized to aspirin in Recovery were not currently on aspirin and had no indication or contra-indication for the use of aspirin, which is in contrast with the observational studies, where there most probably was a non-covid indication for aspirin. This may restrict the conclusions of both the trial and the meta-analysis: patients diagnosed with COVID not currently on aspirin may not benefit from adding aspirin to their treatment, and may have a higher risk of bleeding. Patients on aspirin at the time of diagnosis appear to have a lower risk of dying than patients who were not on aspirin, taking into account various confounders. It would appear preferable not to stop aspirin in patients who have a non-covid indication, but possibly useless to add it if they don't.

Study limitations.

In addition to the limitations inherent in observational studies, or in open-label trials noted above, only English language publications were found and analyzed. Considering the topic and its timeliness, it is unlikely that non-English language publications would alter the results. There may be other English-language studies that were not identified, but it is unlikely that they would modify the results in a relevant manner. The funnel plot does not indicate publication bias. Studies in children were not included, because of negligible mortality (9)

\section{Conclusion}

Aspirin is a widely used antiplatelet drug, and its risks are well known, mainly an increased risk of upper GI bleeding consistent with its irreversible non-selective inhibition of Cox-1, resulting in the anti-platelet activity. From our results and the Recovery trial, it would appear preferable not to stop aspirin in patients who have a non-covid indication, but possibly useless to add it if they don't.

Ethical considerations

This study did not require ethical approval. 
This paper was unfunded.

The authors report performing studies regularly with pharmaceutical industry, required by regulatory authorities, but none concerning COVID-19 and aspirin. They are involved in the surveillance of the COVID19 vaccination program in Europe, with the ACCESS project. The list of these studies can be found atwww.Encepp.eu, in the European PAS registry, and atwww.pharmacoepi.eu.

\section{References}

1. Bianconi V, Violi F, Fallarino F, Pignatelli P, Sahebkar A, Pirro M. Is Acetylsalicylic Acid a Safe and Potentially Useful Choice for Adult Patients with COVID-19 ? Drugs. 2020;80(14):1383-96.

2. Coppola S, Chiumello D. Aspirin in COVID-19 related ARDS: an old, low-cost therapy with a strong rationale. Anesth Analg. 2021.

3. Diaz T, Trachtenberg BH, Abraham SJK, KosagiSharaf R, Durant-Archibold AA. Aspirin Bioactivity for Prevention of Cardiovascular Injury in COVID-19. Front Cardiovasc Med. 2020;7:562708.

4. Salah HM, Mehta JL. Meta-Analysis of the Effect of Aspirin on Mortality in COVID-19. Am J Cardiol. 2021;142:158-9.

5. Martha JW, Pranata R, Lim MA, Wibowo A, Akbar MR. Active prescription of low-dose aspirin during or prior to hospitalization and mortality in COVID-19: A systematic review and meta-analysis of adjusted effect estimates. Int J Infect Dis. 2021;108:6-12.

6. Canzano P, Brambilla M, Porro B, Cosentino N, Tortorici E, Vicini S, et al. Platelet and Endothelial Activation as Potential Mechanisms Behind the Thrombotic Complications of COVID-19 Patients. JACC Basic Transl Sci. 2021.

7. Kevorkian JP, Lopes A, Sene D, Riveline JP, Vandiedonck C, Feron F, et al. Oral corticoid, aspirin, anticoagulant, colchicine, and furosemide to improve the outcome of hospitalized COVID-19 patients - the COCAA-COLA cohort study. J Infect. 2021.

8. Mohamed-Hussein AAR, Aly KME, Ibrahim MAA. Should aspirin be used for prophylaxis of COVID-19induced coagulopathy? Med Hypotheses. 2020;144:109975.

9. Duarte-Salles T, Vizcaya D, Pistillo A, Casajust P, Sena AG, Lai LYH, et al. Baseline characteristics, management, and outcomes of 55,270 children and adolescents diagnosed with COVID-19 and 1,952,693 with influenza in France, Germany, Spain, South Korea and the United States: an international network cohort study. medRxiv. 2020.

10. Viecca M, Radovanovic D, Forleo GB, Santus P. Enhanced platelet inhibition treatment improves hypoxemia in patients with severe Covid-19 and hypercoagulability. A case control, proof of concept study. Pharmacol Res. 2020;158:104950.

11. Chow JH, Khanna AK, Kethireddy S, Yamane D, Levine A, Jackson AM, et al. Aspirin Use is Associated with Decreased Mechanical Ventilation, ICU Admission, and In-Hospital Mortality in Hospitalized Patients with COVID-19. Anesth Analg. 2020.

12. Recovery collaborative group. Aspirin in patients admitted to hospital with COVID-19 (RECOVERY): a randomised, controlled, open-label, platform trial. MedRxiv. 2021.

13. Liu Q, Huang N, Li A, Zhou Y, Liang L, Song X, et al. Effect of low-dose aspirin on mortality and viral duration of the hospitalized adults with COVID-19. Medicine (Baltimore). 2021;100(6):e24544.

14. Meizlish ML, Goshua G, Liu Y, Fine R, Amin K, Chang E, et al. Intermediate-dose anticoagulation, aspirin, and in-hospital mortality in COVID-19: A propensity score-matched analysis. Am J Hematol. 2021;96(4):471-9. 
15. Osborne TF, Veigulis ZP, Arreola DM, Mahajan SM, Roosli E, Curtin CM. Association of mortality and aspirin prescription for COVID-19 patients at the Veterans Health Administration. PLoS One. 2021;16(2):e0246825.

16. Sahai A, Bhandari R, Godwin M, McIntyre T, Chung MK, Iskandar JP, et al. Effect of aspirin on short-term outcomes in hospitalized patients with COVID-19. Vasc Med. 2021:1358863X211012754.

17. Haque S, Jawed A, Akhter N, Dar SA, Khan F, Mandal RK, et al. Acetylsalicylic acid (Aspirin): a potent medicine for preventing COVID-19 deaths caused by thrombosis and pulmonary embolism. Eur Rev Med Pharmacol Sci. 2020;24(18):9244-5.

18. Alamdari NM, Afaghi S, Rahimi FS, Tarki FE, Tavana S, Zali A, et al. Mortality Risk Factors among Hospitalized COVID-19 Patients in a Major Referral Center in Iran. Tohoku J Exp Med. 2020;252(1):73-84.

19. Yuan S, Chen P, Li H, Chen C, Wang F, Wang DW. Mortality and pre-hospitalization use of low-dose aspirin in COVID-19 patients with coronary artery disease. J Cell Mol Med. 2021;25(2):1263-73.

20. Martha JW, Pranata R, Lim MA, Wibowo A, Akbar MR. Active Prescription of Low-dose Aspirin During or Prior to Hospitalization and Mortality in COVID-19 - A Systematic Review and Meta-analysis of Adjusted Effect Estimates. Int J Infect Dis. 2021.

21. Merzon E, Green I, Vinker S, Golan-Cohen A, Gorohovski A, Avramovich E, et al. The use of aspirin for primary prevention of cardiovascular disease is associated with a lower likelihood of COVID-19 infection. FEBS J. 2021.

22. Meizlish ML, Goshua G, Liu Y, Fine R, Amin K, Chang E, et al. Intermediate-dose anticoagulation, aspirin, and in-hospital mortality in COVID-19: a propensity score-matched analysis. medRxiv. 2021.

Legends to the figures

Figure 1 meta-analysis of aspirin and COVID-19 associated deaths; Inverse variance with random effects model.

Figure 2 meta-analysis of aspirin and COVID-19 associated deaths; Inverse variance with fixed effects model.

Figure 3 meta-analysis of aspirin in COVID-19 associated deaths, excluding the Recovery trial. Inverse variance with random effects model.

Figure 4: funnel plot for meta-analysis of studies of COVID-19 deaths in patients exposed to aspirin.

Table 1 Search results on June 7, 2021

COVID in TITLE 98155

AND

Aspirin (Title) 28

Aspirin (all fields) 87

AND (Death or mortality) 12

Studies with abstract and results 8

Table 2

Study first author

\begin{tabular}{lllllll}
\hline Study author & Study type & Controls & Events & Exposed & Exposed Events & OR* [95\%CI] \\
\hline Alamdari (18) & C+, Adj & 459 & 63 & 53 & 9 & $1.23[0.58-2.61]$ \\
Chow (11) & C+, Adj & 314 & 73 & 98 & 26 & $0.53[0.31-0,90]$
\end{tabular}




\begin{tabular}{lllllll}
\hline Study author & Study type & Controls & Events & Exposed & Exposed Events & OR $^{*}[95 \% \mathrm{CI}]$ \\
\hline Liu (13) & C+, PS-M & 24 & 10 & 24 & 1 & $0.19[0.05-0.78]$ \\
Meizlich (22) & C+, PS-M & 319 & N/A & 319 & N/A & $0.52[0.34-0.81]$ \\
Merzon (21) & C+ Adj & 112 & 7 & 21 & 1 & $0.36[0.02-0.85]$ \\
Osborne (15) & C+ PS-M & 6814 & 437 & 6814 & 170 & $0.38[0.32-0.46]$ \\
Recovery (12) & C+ open RCT & 7541 & 1299 & 7351 & 1222 & $0.96[0.89-1.04]$ \\
Sahai (16) & C+ PS-M & 248 & 38 & 248 & 33 & $0.85[0.51-1.41]$ \\
Yuan (19) & C+ Adj & 131 & 29 & 52 & 11 & $0.94[0.41-2.17]$ \\
\hline
\end{tabular}

C+ Patients positive for Covid-19; Adj: adjusted cohort study; PS-M propensity score matched cohorts; $\mathrm{RCT}$ randomized clinical trial

* Odds ratios as reported in publications

N/A numbers not provided.

\section{Hosted file}

image1.emf available at https://authorea.com/users/432015/articles/535489-aspirin-and-deathin-covid-19-a-systematic-review-and-meta-analysis

Figure 1 Forest plot of aspirin and COVID-19 associated deaths; Inverse variance with random effects model.

\section{Hosted file}

image2.emf available at https://authorea.com/users/432015/articles/535489-aspirin-and-deathin-covid-19-a-systematic-review-and-meta-analysis

Figure 2 Forest plot of aspirin and COVID-19 associated deaths; Inverse variance with fixed effects model.

\section{Hosted file}

image3.emf available at https://authorea.com/users/432015/articles/535489-aspirin-and-deathin-covid-19-a-systematic-review-and-meta-analysis

Figure 3 Forest Plot of aspirin in COVID-19 associated deaths, excluding the Recovery trial (12). Inverse variance with random effects model.

\section{Hosted file}

image4.emf available at https://authorea.com/users/432015/articles/535489-aspirin-and-deathin-covid-19-a-systematic-review-and-meta-analysis

Figure 4: Forest Plot of aspirin in COVID-19 associated deaths, excluding the two largest studies, Recovery (12) and Osborne (15). Inverse variance with random effects model.

\section{Hosted file}

image5.emf available at https://authorea.com/users/432015/articles/535489-aspirin-and-deathin-covid-19-a-systematic-review-and-meta-analysis

Figure 5: funnel plot for meta-analysis of studies of COVID-19 deaths in patients exposed to aspirin. 